\title{
Thermal neutron scattering evaluation framework
}

\author{
Chris Chapman ${ }^{1, a}$, Luiz Leal ${ }^{2}$, Farzad Rahnema ${ }^{1}$, Yaron Danon ${ }^{3}$, and Goran Arbanas ${ }^{4}$ \\ 1 Georgia Inst Technol, 770 State St NW, Room 3-39S, Atlanta, GA 30318, USA \\ 2 PSN-EXP/SNC/LNR, IRSN, Fontenay-aux-Roses, France \\ 3 Rensselaer Polytech Inst, Gaerttner LINAC Ctr, Troy, NY 12180, USA \\ 4 Oak Ridge Natl Lab, Reactor \& Nucl Syst Div, Oak Ridge, TN 37831, USA
}

\begin{abstract}
A neutron scattering kernel data evaluation framework for computation of model-dependent predictions and their uncertainties is outlined. In this framework, model parameters are fitted to doubledifferential cross section measurements and their uncertainties. For convenience, the initial implementation of this framework uses the molecular dynamics model implemented in the GROMACS code. It is applied to light water using the TIP4P/2005f interaction model. These trajectories computed by GROMACS are then processed using nMOLDYN to compute the density of states, which is then used to calculate the scattering kernel using the Gaussian approximation. Double differential cross sections computed from the scattering kernel are then fitted to double-differential scattering data measured at the Spallation Neutron Source detector at Oak Ridge National Laboratory. The fitting procedure is designed to yield optimized model-parameters and their uncertainties in the form of a covariance matrix, from which new evaluations of thermal neutron scattering kernel will be generated. The Unified Monte Carlo method will be used to fit the simulation data to the experimental data.
\end{abstract}

\section{Introduction}

With the rise in interest of GEN-IV reactor systems, specifically very high temperature and molten salt reactors, there has been a need for newer, more accurate thermal scattering nuclear data. In addition to GEN-IV reactors, current light water reactors that are applying for license extensions need high fidelity cross sections and uncertainties to better quantify whether they can operate safely for another 20 years. In addition, thermal moderator data plays a key role in nuclear criticality safety analyses. Currently, there are very limited thermal moderator data for materials that are of interest to nuclear criticality safety (e.g., lucite, paraffin, hydrofluoric acid, etc.). The lack of uncertainties or covariance data for thermal scattering material means there is no way of quantifying the effects of thermal scattering uncertainties in quantities of interest in reactor systems.

The framework outlined here aims to alleviate these issues by providing a generalized method to generate thermal double differential scattering cross sections and

\footnotetext{
a e-mail: c86@ornl.gov

This manuscript has been authored by UT-Battelle, LLC under Contract No. DE-AC05-00OR22725 with the U.S. Department of Energy. The United States Government retains and the publisher, by accepting the article for publication, acknowledges that the United States Government retains a non-exclusive, paidup, irrevocable, world-wide license to publish or reproduce the published form of this manuscript, or allow others to do so, for United States Government purposes. The Department of Energy will provide public access to these results of federally sponsored research in accordance with the DOE Public Access Plan (http://energy.gov/downloads/doe-public-access-plan).
}

their respective uncertainties and covariances from model parameters. This is done by fitting the computer simulated scattering data against experimental data using the Unified Monte Carlo (UMC) method, which had previously only been used on fast spectrum data. This method will be independent of how the simulations are run (classical molecular dynamics, ab-initio, quantum mechanics, etc.), and how the structure factor is generated, which currently does not exist. These new cross sections will then be validated against experimental benchmarks as well as other experimentally gathered data.

\section{Double differential cross section}

Neutron cross sections can be categorized into three energy regions: thermal, epithermal, and fast. In the epithermal and fast energy regions, the neutron is energetic enough to render the vibrational energy of the target nucleus as well as the binding energies of a target molecular or crystalline structure as negligible. In the thermal region, however, the neutron energy is comparable to these vibrational and binding energies, meaning they have to be taken into account when considering what the neutron cross section is at these energies.

Thermal scattering cross sections can be divided into two components: coherent scattering and incoherent scattering. Coherent scattering occurs when neutrons scatter with different nuclei and their wavefunctions constructively interfere with one another. It is more prevalent in solids, such as graphite, and materials with a crystalline lattice structure, though any material with a non-negligible coherent cross section may exhibit significant coherent scattering. Incoherent scattering 
occurs when there is no interference between scattering neutron wavefunctions and is more noticeable in liquids and hydrogenous materials like water.

The double differential cross section in the thermal energy region is calculated from the scattering law, as shown in [1], which is calculated from the intermediate structure factor. Traditionally, the intermediate structure factor has been calculated one of two ways: the Van Hove Theory (VHT), as shown below,

$$
\begin{aligned}
& F_{V H T}(\vec{q}, t)=\sum_{\alpha, \beta} \Gamma_{\alpha \beta}\left\langle e^{-i \vec{q} \cdot \hat{R}_{\alpha}(0)} e^{i \vec{q} \cdot \hat{R}_{\beta}(t)}\right\rangle, \\
& \Gamma_{\alpha \beta}=\frac{1}{N}\left(\overline{b_{\alpha}} \overline{b_{\beta}}-\delta_{\alpha \beta}\left(\overline{b_{\alpha}^{2}}-{\overline{b_{\alpha}}}^{2}\right)\right),
\end{aligned}
$$

or the Gaussian approximation with density of states (DoS), as shown below,

$$
\begin{aligned}
& F_{D o S}(q, t)=\exp \left[-\frac{q^{2}}{2} \frac{\hbar}{m} \int_{0}^{\infty} d \omega \frac{\rho(\omega)}{\omega}\right. \\
& \left.\left.\times\left\{\operatorname{coth}\left(\frac{\hbar \omega}{2 k_{B} T}\right)(1-\cos (\omega t))-i \sin \omega t\right)\right\}\right],
\end{aligned}
$$

where $\rho(\omega)$ is the density of states, $\hat{R}_{\alpha}(t)$ is the quantum mechanical position operator of particle $\alpha$ at time $t$, and $b_{\alpha}$ is the scattering length of particle $\alpha$.

The VHT (Eqs. (1) \& (2)) states that the intermediate structure factor is represented by a quantum thermal average of the position of the atoms. The time dependence of the position operator is defined by the Heisenberg picture of quantum mechanics. The first term in Eq. (2) accounts for the coherent scattering, while the second term accounts for incoherent scattering. In the classical framework, the position operator is replaced by the timedependent position vector of the particles, more commonly known as the trajectories of the particles, and the quantum thermal averages are replaced by classical ensemble averages. Recent work using the VHT has been done by the NAUSICAA collaboration [2].

The Gaussian approximation (Eq. (3)) assumes that the scattering occurs in a medium with isotropic scattering. With this assumption, the intermediate structure factor is represented by a Gaussian as a function of the density of states (also known as the frequency spectrum). This is the more widely used method and is currently used by NJOY to calculate scattering data for MCNP [3].

The VHT can clearly be used to calculate both coherent and incoherent scattering, while the Gaussian approximation (in its current formalism) can only be applied to incoherent scattering. There are methods for approximating the coherent component in systems, such as the Vineyard and Sköld approximation [4]. The drawback to the VHT is that, by viewing it in the classical framework, the probabilities are equal for up-scattering and downscattering, which is non-physical. There are several semiclassical approximations that can be applied, such as the Harmonic, Schofield, Schofield-Harmonic, and the "Standard" approximations [5].

\section{Unified Monte Carlo}

The UMC approach is based on Bayes Theorem and the Principle of Maximum Entropy [6] and was first proposed by Smith [7]. There are currently two approaches that, while they both have a similar underlying mathematical formalism, differ slightly in their application: UMC-G and UMC-B [8].

\subsection{UMC-G}

If $\mathbf{y}_{E}$ is the experimental dataset containing $m$ values, $\mathbf{V}_{E}$ is the experimental covariance matrix, $\boldsymbol{x}_{C}$ is the computed dataset containing $n$ values, and $\mathbf{V}_{C}$ is the computed covariance matrix, then Bayes Theorem states that

$$
p(\boldsymbol{x})=\mathcal{N} L\left(\mathbf{y}_{E}, \mathbf{V}_{E} \mid \boldsymbol{x}\right) p_{o}\left(\boldsymbol{x} \mid \boldsymbol{x}_{C}, \mathbf{V}_{C}\right),
$$

where $p(x)$ is the posterior probability density function (PDF), $p_{o}$ is the prior PDF, and $L$ is the likelihood PDF. The normalization constant $\mathcal{N}$ is included to ensure that the posterior PDF integrates to unity. In this framework, $\mathbf{y}_{E}$ would be the measured double differential cross section, and $\mathbf{V}_{E}$ would be its associated covariance. The vector $\boldsymbol{x}$ contains $m$ random variables, and are arguments of the posterior PDF $p$. The mean value of this random variable vector $\boldsymbol{x}$, weighted by the posterior PDF, and its associated covariance matrix are defined as

$$
\begin{aligned}
& \left\langle x_{i}\right\rangle=\int x_{i} p(\boldsymbol{x}) d \boldsymbol{x}, \\
& (\mathbf{V})_{i, j}=\left\langle x_{i} x_{j}\right\rangle-\left\langle x_{i}\right\rangle\left\langle x_{j}\right\rangle .
\end{aligned}
$$

Using the Principle of Maximum Entropy, the optimal choice for the PDF is a multivariate Gaussian function, which leads to Eq. (4) being recast as

$$
\begin{aligned}
p(\boldsymbol{x}) \sim \exp \left\{-\frac{1}{2}\right. & {\left[\left\{\left(\mathbf{y}-\mathbf{y}_{E}\right)^{T} \cdot \mathbf{V}_{E}^{-1} \cdot\left(\mathbf{y}-\mathbf{y}_{E}\right)\right\}\right.} \\
+ & \left.\left.\left\{\left(\boldsymbol{x}-\boldsymbol{x}_{C}\right)^{T} \cdot \mathbf{V}_{C}^{-1} \cdot\left(\boldsymbol{x}-\boldsymbol{x}_{C}\right)\right\}\right]\right\}
\end{aligned}
$$

In this framework, $\mathbf{y}_{E}$ is the measured double differential cross section, and $\boldsymbol{x}_{C}$ is the calculated dynamic structure factor. The variable $\mathbf{y}$ is therefore defined as $\mathbf{y}=\mathbf{f}(\boldsymbol{x})$, where $\mathbf{f}$ contains $n$ scalar functions, each of whose variables are one or more of the elements of the random variable $\boldsymbol{x}$. With this in mind, the integrals of Eqs. (5) and (6) can be rewritten as

$$
\begin{aligned}
& \left\langle x_{i}\right\rangle=\lim _{K \rightarrow \infty} \frac{\sum_{k=1}^{K} x_{i k} p\left(\boldsymbol{x}_{k}\right)}{\sum_{k=1}^{K} p\left(\boldsymbol{x}_{k}\right)} \\
& (\mathbf{V})_{i, j}=\lim _{K \rightarrow \infty} \frac{\sum_{k=1}^{K} x_{i k} x_{j k} p\left(\boldsymbol{x}_{k}\right)}{\sum_{k=1}^{K} p\left(\boldsymbol{x}_{k}\right)}-\left\langle x_{i}\right\rangle\left\langle x_{j}\right\rangle .
\end{aligned}
$$

From here, the random variable $\boldsymbol{x}_{k}$ is sampled using a Monte Carlo sampling scheme including a brute force method, or a Metropolis algorithm.

\subsection{UMC-B}

The UMC-B formulation, like UMC-G, is founded on the ideals of Bayes Theorem and the Principle of Maximum Entropy. Unlike in UMC-G, however, the mean values of the simulation data $\boldsymbol{x}_{C}$ and $\mathbf{V}_{C}$ are not calculated. Rather, 


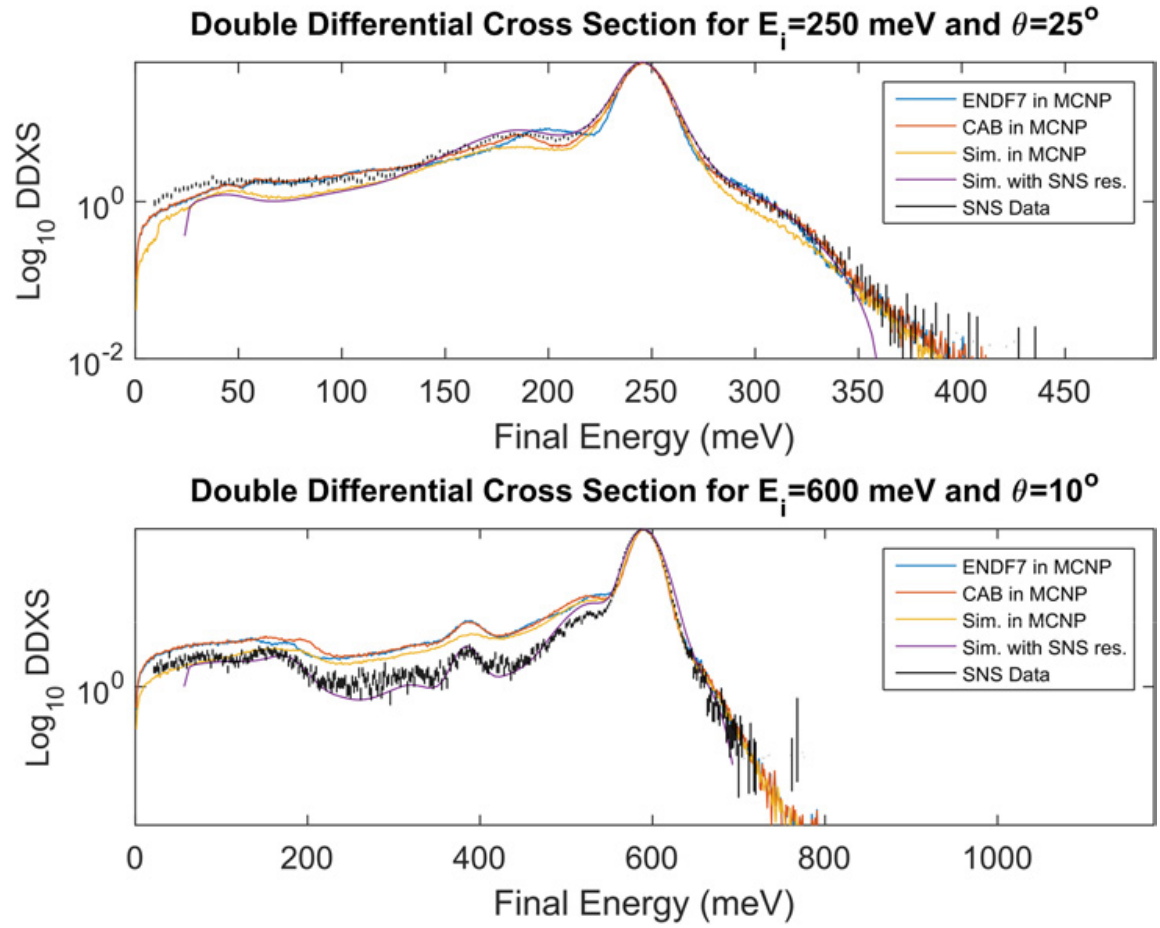

Figure 1. Double differential scattering cross section for light water at $250 \mathrm{meV}$ at $25^{\circ}$ and $600 \mathrm{meV}$ at $10^{\circ}$.

a collection of scalar weighting values $\omega_{k}$ are calculated where, for each simulation $k$,

$$
\omega_{k}=\exp \left\{-\frac{1}{2}\left[\left(\mathbf{y}_{k}-\mathbf{y}_{E}\right)^{T} \cdot \mathbf{V}_{E}^{-1} \cdot\left(\mathbf{y}_{k}-\mathbf{y}_{E}\right)\right]\right\} \text {. }
$$

As in the UMC-G case, $\mathbf{y}_{k}=\mathbf{f}\left(\boldsymbol{x}_{C k}\right)$, where $\boldsymbol{x}_{C k}$ is calculated using model parameters sampled using the same prior distribution as in UMC-G. The information of the prior and likelihood functions are combined into one function, like in the UMC-G case.

The weighting value $\omega_{k}$ is a measure of the deviation between the experimental data $\mathbf{y}_{E}$ from the simulation data $\mathbf{y}_{k}$ for the $k^{\text {th }}$ simulation history. Once the simulation values $\mathbf{y}_{k}$ and $\omega_{k}$ are calculated, the mean values $\boldsymbol{x}$ and covariance matrix $\mathbf{V}$ can be calculated using

$$
\begin{aligned}
& \left\langle x_{i}\right\rangle=\lim _{K \rightarrow \infty} \frac{\sum_{k=1}^{K} x_{i k} \omega_{k}}{\sum_{k=1}^{K} \omega_{k}}, \\
& (\mathbf{V})_{i, j}=\lim _{K \rightarrow \infty} \frac{\sum_{k=1}^{K} x_{i k} x_{j k} \omega_{k}}{\sum_{k=1}^{K} \omega_{k}}-\left\langle x_{i}\right\rangle\left\langle x_{j}\right\rangle .
\end{aligned}
$$

One advantage of UMC-B over UMC-G is that the covariance matrix of the simulation data $\mathbf{V}_{C}$ does not need to be calculated or inverted. This is advantageous to its applicability to thermal scattering data, as $S(\alpha, \beta)$ scattering laws have been as large as [287 $\times 187]$, which would lead to a $\mathbf{V}_{C}$ of size [53669 $\left.\times 53669\right]$. One significant downside of UMC-B, however, is that there is no apparent way to "speed up" the Monte Carlo sampling as one can do in UMC-G by using the Metropolis algorithm [7].

\section{Preliminary results}

To prove the validity of this framework, it is being tested out on light water. Experimental data were collected from the ORNL Spallation Neutron Source (SNS) at 55, 160, $250,600,1000,3000, \& 5000 \mathrm{meV}$ between $3^{\circ}$ and $58^{\circ}$ with $1^{\circ}$ increments. A system of 343 light water molecules were modeled in the molecular dynamics (MD) code GROMACS [9]. The molecules were parameterized using the TIP4P/2005f interaction potential [10]. The simulation was then run for 100 ps with a timestep of 0.1 fs at constant volume and temperature. This leads to a $\Delta E$ of $0.05 \mathrm{meV}$ and an $E_{\text {max }}$ of $10 \mathrm{meV}$. Trajectories from the GROMACS run were then post-processed using nMOLDYN [11] to generate density of states. These density of states are then used to calculate the intermediate structure factor. Due to the need for energies above 50 $\mathrm{meV}$, the Gaussian approximation is used to calculate the intermediate structure factor. It is then convoluted with the SNS detector resolution function so it can be compared to the experimental data.

A plot of the double differential cross section with the detector resolution function applied is shown in Fig. 1. As a comparison, the SNS data is plotted alongside the simulation data along with MCNP simulations designed to replicate the SNS measurements run with three different cross section sets: the cross sections found in ENDF7 [12], the cross sections from the CAB model [13], and the simulation cross sections which were processed by NJOY using the simulated density of states and read into MCNP. Specifically, the density of states was processed by NJOY in the same manner that was used to process the ENDF/B-VII thermal scattering cross section. In the MCNP runs, a Gaussian distribution of the source particles is assumed, which is slightly less accurate than the true SNS simulation but is a sufficient approximation. It is worth noting that, since these are preliminary results, no UMC calculation has been carried out, and as such, there are no uncertainties of the double differential cross section to plot. Additionally, a plot of the total cross section is shown in Fig. 2. The total scattering cross section agrees 


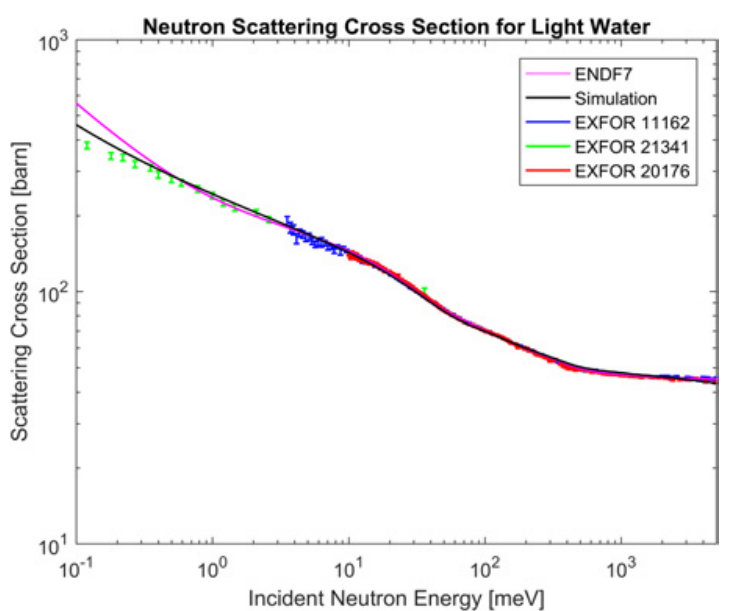

Figure 2. Total neutron scattering cross section for light water.

very favorably with both the ENDF/B-VII data as well as the EXFOR data [14], and actually has an improved agreement with the EXFOR data between 0.1-1 meV.

\section{Conclusion \& future work}

A framework for generating thermal scattering cross sections and their covariances is laid out. While experimental data are not required, its inclusion will greatly improve the accuracy of the framework results. There is still a great deal of work to be done before this task is completed. The exact specifications of the MD simulation have not be finalized yet, meaning UMC has not been applied to generate the new dataset. The framework, which aims to fit simulation data and experimental data using the Unified Monte Carlo method, will be applied to light water.

There may be ways to improve upon the VHT, such that it could extend more formally to the quantum framework instead of being limited to the classical framework. Such an improvement could yield more accurate results than the Gaussian approximation. There has been a recent attempt to combine the Gaussian approximation with the VHT [15], which could be expanded to include coherent contributions. There currently does not exist a method to propagate thermal scattering covariance data through neutron transport codes. This is most likely due to the current lack of thermal scattering covariance data, but such functionality would encourage the community to look into other innovative methods for generating thermal scattering covariance data. In addition, the framework can, in theory, be applied to any material, and it is will be applied to other thermal materials for reactor applications, such as graphite, lucite, or teflon.
The first author would like to acknowledge the support provided by the U.S. Department of Energy Nuclear Energy University Program Graduate Fellowship. He would also like to thank Kemal Ramic and Carl Wendorff of RPI for their help in providing the data, as well as Garrett Granroth and Alexander Kolesnikov of the ORNL SNS for their assistance in providing the SNS resolution function. This research used resources of the National Energy Research Scientific Computing Center, a DOE Office of Science User Facility supported by the Office of Science of the U.S. Department of Energy under Contract No. DE-AC02$05 \mathrm{CH} 11231$. The work by the 4th and 5th authors was supported by the DOE Nuclear Criticality Safety Program, funded and managed by the National Nuclear Security Administration for DOE.

\section{References}

[1] Squires, G.L., Thermal Neutron Scattering (Cambridge University Press, Cambridge, 1978)

[2] Farhi, E., Ferran, G., Haeck, W., Pellegrini, E., Calzavara, Y. J. Nucl. Sci. Technol. 52, 844-856 (2015)

[3] MacFarlane, R.E., Muir, D.W. "The NJOY Nuclear Data Processing System", LA-12740-M (1994)

[4] Mattes, M., Keinert, J., INDC/NDS report INDC(NDS)-0470 (April 2005)

[5] Valleau, S., Eisfeld, A., Aspuru-Guzik, A., The Journal of Chemical Physics 137, 224103 (2012)

[6] Smith, D.L., Probability, Statistics and Data Uncertainties in Nuclear Science and Technology (American Nuclear Society LaGrange Park, IL USA, 1991)

[7] Capote, R., Smith, D.L., Nuclear Data Sheets 109, 2768-2773 (2008)

[8] Capote, R., Smith, D.L., Trkov, A., Meghzifene, M., Journal of ASTM International 9 (2012)

[9] Van Der Spoel, D., Lindahl, E., Hess, B., Groenhof, G., Mark, A.E., Berendsen, H.J.C., Journal of Computational Chemistry 26, 1701-1718 (2005)

[10] González, M.A., Abascal, J.L.F., The Journal of Chemical Physics 135, 224516 (2011)

[11] Hinsen, K., Pellegrini, E., Stachura, S., Kneller, G.R., Journal of Comp. Chem. 33, 2043-2048 (2012)

[12] Chadwick, M.B., Herman, M., Obložinský, P., Dunn, M.E., Danon, Y., Kahler, AC., Smith, DL., Pritychenko, B., Arbanas, G., Arcilla, R., et al., Nuclear Data Sheets 112, 2887 (2011)

[13] Marquez Damian, J.I., Malaspina, D., Granada, J.R., Ann. Nucl. Energy 65, 280 (2014)

[14] N. Otuka, E. Dupont, V. Semkova et al., Nuclear Data Sheets 120, 272-276 (2014)

[15] Abe, Y., Tasaki, S., Ann. Nucl. Energy 83, 302-308 (2015) 